\title{
Possible Relationship between Long Term Use of Cell Phones and Tumors
}

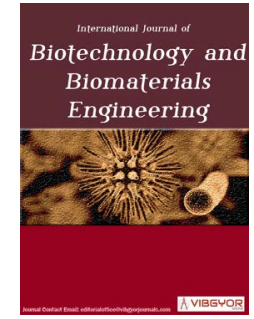

\section{Khaari Beaubrun, Kieviyonnia Ward, Anita Mandal, Victoria Raade, Makeyla Hickmon, Jarett Raade and Prabir Mandal'}

Biology Program, Department of Mathematics \& Sciences, Edward Waters College, USA

\begin{abstract}
Cell (or cellular or mobile) phones first became widely available in the United States in the 1990s, but their use has increased dramatically since then. Cell phone use is nowadays an unremarkable part of everyday activities. Cell phones use radio-waves to carry information between callers. The frequency of these waves ranges from about 850-1900 megahertz ( $\mathrm{MHz}$ ). The higher the frequencies, the greater the energy carried by the waves. Human brain cells communicate via electrical impulses, which can be detected by non-invasive EEG (electroencephalogram) measurements. Positron Emission Tomography (PET) imaging is another tool used to study the effect of cell phone radiation on human brain activity. With nearly 300 million cell phones in use in the United States and about 6.8 billion mobile phone subscription are active at present throughout the world, some experts say the concern lies with not only with the long-term impact on healthy individuals but in consumers with unhealthy cells as well. The dramatic increase in the use of cell phones has generated concerns about potential adverse effects, especially the development of brain tumors.
\end{abstract}

Keywords

Cell phone, Radiation, Glioma, Electromagnetic field, Radio frequency, Epidemiology

\section{Introduction}

Cell phones emit a type of radiation that is known as Radio Frequency-Electromagnetic radiation Fields (RF-EMF) energy, also referred to as microwave radiation, so some concerns have been raised about the safety of cell phone use. Cell phones work by sending Universal Mobile Telecommunication System (UMTS) signals to nearby cell towers using RF waves. This is a form of electromagnetic energy that falls between FM radio waves and microwaves. Like FM radio waves, microwaves, visible light, and heat, RF waves are a form of non-ionizing radiation. Many factors can affect the amount of RF energy to which a person is exposed e.g., whether the person is using the speaker mode on the phone or a hands-free device, the amount of time the person is on phone, the model of the phone being used, the distance and path to the nearest cell phone tower, the amount of cell phone traffic in the area at the time etc. The amount of the RF energy absorbed from the phone into the user's body is known as the Specific Absorption Rate (SAR). Different cell phones have

\footnotetext{
*Corresponding author: Dr. Prabir K Mandal, Professor \& Interim Chair, Biology Program, Department of Mathematics \& Sciences, Edward Waters College, 1658 Kings Road, Jacksonville, FL 32209, USA, Tel: (904)-470-8091

Accepted: November 27, 2018; Published: November 29, 2018

Copyright: @ 2018 Beaubrun K, et al. This is an open-access article distributed under the terms of the Creative Commons Attribution License, which permits unrestricted use, distribution, and reproduction in any medium, provided the original author and source are credited.

Beaubrun et al. Int J Biotechnol Biomater Eng 2018, 1:001
} 
different SAR levels. Cell phone makers are required to report the maximum SAR level of their product to the US Federal Communications Commission (FCC). The upper limit of SAR allowed in the United States is 1.6 watts per kilogram of body weight. The actual SAR value during use varies based on a number of factors, so it's possible that a phone with a lower listed SAR value might actually expose a person to more RF energy than one with a higher listed SAR value in some cases. Bluetooth earpieces have an SAR value of around 0.001 watts $/ \mathrm{kg}$. The World Health Organization (WHO) and International Agency for Research on Cancer (IARC) in 2011 classified, based on the scientific evidence available at the time, cell phone radiation (frequency range $30 \mathrm{KHz}-300 \mathrm{GHz}$ ) as a "possible human carcinogen" due to an increased risk of brain cancer from longterm and heavy use of cell phones [1,2].

\section{Reports of Cell Phone Usage and its Effects}

Because cell phones usually are held near the head when being used, the main concern has been whether they might cause or contribute to tumors in the areas viz., malignant brain tumors such as gliomas, non-cancerous tumors of the brain such as meningioma, non-cancerous tumors of the nerve connecting the brain to the ear such as vestibular schwannomas (also known as acoustic neuromas) and non-cancerous tumors of the salivary glands. Eighty percent $(80 \%)$ of the absorbed radiation comes from holding the phone to the head. Interestingly, they brain's memory function is more vulnerable to the negative impact of radiation when the phone is held to the right side of the head. That's where the areas of the brain related to memory are located. Using your cell phone a half hour per day increases your risk of brain tumor by $40 \%$ [3].

Epidemiologic studies have been a primary method of research on if cell phones cause cancer and more specifically the effects of radiofrequency energy exposure. Another study have investigated the correlation of cell phone use and risk of both malignant (e.g., gliomas) and benign (e.g., acoustic neuromas) brain tumors, meningioma, parotid gland and salivary gland tumors. Bortkiewicz, et al. reported the hypothesis that long-term use of mobile phone increased risk of intracranial tumors, especially in the case of ipsilateral exposure [4]. According to the NIH National Institute on Deafness and Other Communication Disorders
(NIDCD), these types of studies look at the factors that determine how many people have a disease or disorder by examining factors (how time, participants understanding of a question (asked verbally, in a questionnaire or, otherwise asked), effects on culture, and effects on economy) that would affect the research data collected. Another study looking at radiofrequency energy exposure gather information from several sources including questionnaires and data from cell phone service providers according to the NIH National Cancer Institute. Also, in accordance to the NIH National Cancer Institute, out of laboratory research estimates consider many factors such as frequency (looking at number of calls daily, weekly, or monthly, how long participants have used phones over there lifetime and what age they started using cell phones, average duration of typical cell phone call, the total hours of lifetime use [5].

A study conducted by Larjavaara, Schüz, Swerdlow, et al. [5] titled Location of gliomas in relation to mobile telephone use: $A$ case-case and case-specular analysis is considered to be the largest study of cell phone use and risk of head and neck tumors. The authors considered the amount of usage of phones by participants included in the study as well as where the participants placement of phone when in use (left, right, or both sides of the head) in relation to the location of the tumor. There were two different scientific approaches taken by the authors. Tumor locations were compared with varying exposure levels in the case-case analysis approach. In the second approach case-specular analysis a hypothetical reference location was assigned for each glioma, and the distances from the actual and hypothetical (specular) locations were compared. The study participants were all glioma diagnosed patients that met study inclusion criteria from 7 different countries including Denmark, Finland, Germany, Italy, Norway, Sweden, and England. The studied period was between 2000 and 2004 and 888 cases were included in the study. The overall results of the study did not fulfill the hypothesis of gliomas among mobile phone users being preferentially located in the parts of the brain with the highest radio-frequency exposure.

"In the case-case analyses, gliomas among contralateral and never-regular users, representing lower radio-frequency exposures, had a shorter mean distance between the tumor midpoint and 
the presumed source of exposure than ipsilateral and regular users. In the case-specular analysis, both exposed and unexposed glioma cases had tumors non-significantly located within $5 \mathrm{~cm}$ from the phone more frequently than the hypothetical locations assigned for specular, but no such pattern was found in analyses by amount of phone use. In both models, glioma cases were closer to the exposure line in long-term users, but the differences remained nonsignificant" [6]. A conclusion was met that the studies as a whole showed no statistical significance that there is a correlation between cell phone use and risk of head and neck tumors or cancer.

A small government funded study published in the Journal of American Medical Association is the first to look specifically at how electromagnetic radiation from cell phones affects glucose metabolism indicated that exposure to cell phones activate the brain much more easily than previously thought. Brain activity means that the cells are using glucose to create energy. When glucose metabolism goes up, it activates cells [7-10]. The National Toxicology Program reported that RF EMF exposures significantly increase gliomas (brain) and schwannomas (heart) [10]. The induction of cancer is thought to occur largely by the initiation of DNA lesions. lonizing radiation, which is known to induce brain cancer, is thought to do so by initiating large scale chromosomal damage resulting from DNA breakage and rearrangement [11,12]. Epidemiologic evidence compiled in the past 20 years starts to indicate risk, particularly for brain tumors (glioma, meningioma, acoustic neuroma), from use of cellular phone. Quickly changing technologies and intensive uses of radiofrequency electromagnetic field-emitting phones pose a challenge to public health. Cell phone users and uses and exposures to other Wireless Transmitting Devices (WTDs) have increased in the past few years [13].

\section{Discussion}

With the increasing use of cell phones worldwide, Electromagnetic Fields (EMF) exposure from cell phones has become a serious potential public health problem including the annual incidence of certain types of brain cancers. For several decades, researchers have been examining the correlation between the use of cellphones and cancer risk. There are several reasons as to why this has become a concern. The main reason is that cellphones emit radiofrequency energy. If someone already has brain damage and then stimulates it with electromagnetic exposure from a cell phone, it could trigger something dangerous. An even greater increased risk has been suggested for children due to thinner skull, smaller head and increased brain conductivity. Children's cells divide at a faster rate, so the impact of radiation can be much larger, which is why we believe the pediatric population is at a higher risk. While waves of higher energies can heat living tissues enough to cause damage, the heat generated by cell phones is so small that few scientists believe they can do any damage to human users. Some studies have found that brain tumor patients who use cell phones have tumors that grow faster than tumors in those who don't use cell phones. Analog (NMT) cell phones operate on a different frequency than digital (GSM) cell phones. A study suggested that users of analog cell phones had a slightly greater risk of developing brain tumors than did nonusers of cell phones, and the risk increased for each year of use. A study using software modified mobile phones recording operating characteristics found that the average output power was approximately $50 \%$ of the maximum power level for digital phones. Children that use cell phones more and spend a longer amount of time are more likely to have AttentionDeficit/Hyperactivity Disorder (ADHD) [14]. Cell phone use is in agreement with the hypothesized spatial and temporal associations, is related to an increased risk of developing a malignant neoplasm in the temporal lobe.

As for other smartphone uses viz., sending text message, taking photos, using apps almost no radiation exposure to the brain. If you are concerned, the scientist points out that the radiation effect can easily be minimized by taking calls on the left side of your head, or using speakerphone or headphone especially when the network quality is low. The precautionary tips on how to protect your children from the health issues that could be connected to cell phone radiation are decrease use of phones or WiFi, turn off wireless networks and devices, turn airplane mode on when you are not using its wireless functions, use of speaker phone or a plug in earpiece/airtube headset (Bluetooth headset also emits radiation), avoid carrying your cell phone in your pocket or anywhere on your body when it's switched on, and increase the distance between cell phone and users. 


\section{References}

1. Baan R, Grosse $Y$, Lauby-Secretan B, El Ghissassi F, Bouvard V, et al. (2011) Carcinogenicity of radiofrequency electromagnetic fields. Lancet Oncol 12: 624-626.

2. IARC Monographs on the Evaluation of Carcinogenic Risks to Humans (2013) Non-lonizing Radiation, Part 2: Radiofrequency Electromagnetic Fields. International Agency for Research on Cancer, Lyon, France.

3. https://thetruthaboutcancer.com/cell-phoneradiation-cancer/

4. Bortkiewicz A, Gadzicka E, Szymczak W (2017) Mobile phone use and risk for intracranial tumors and salivary gland tumors - A meta-analysis. Int J Occup Med Environ Health 30: 27-43.

5. Larjavaara S, Schüz J, Swerdlow A, Feychting $M$, Johansen C, et al. (2011) Location of gliomas in relation to mobile telephone use: $\mathrm{A}$ case-case and case-specular analysis. Am J Epidemiol 174: 2-11.

6. Wang P, Hou C, Li Y, Zhou D (2018) Wireless phone use and risk of adult glioma: Evidence from a meta- analysis. World Neurosurg 115: e629-e636.

7. Linet MS, Inskip PD (2010) Cellular (mobile) telephone use and cancer risk. Rev Environ Health 25: 51-55.

8. (2018) Cell phones and cancer risk.

9. (2018) Cell phones - Radiofrequency background.

10.(2018) Cell phone radio frequency radiation.

11.United Nations Scientific Committee on the Effects of Atomic Radiation (UNSCEAR) (2008) Annex A: Epidemiological Studies of radiation and cancer. United Nations.

12.Little MP, Wakeford R, Tawn EJ, Bouffler SD, Berrington de Gonzalez A (2009) Risks associated with low doses and low dose rates of ionizing radiation: Why linearity may be (almost) the best we can do. Radiology 251: 6-12.

13.Morgan LL, Miller AB, Sasco A, Davis DL (2015) Mobile phone radiation causes brain tumors and should be classified as a probable human carcinogen (2A) (review). Int J Oncol 46: 1865-1871.

14.https://www.health.harvard.edu/blog/can-cellphone-use-cause-adhd-2018073114375 